\title{
Indicadores socioeconômicos e serviços odontológicos em uma região brasileira desfavorecida $^{1}$
}

\author{
Andréa Clemente Palmier, ${ }^{2}$ Danielle Alves Andrade, ${ }^{3}$ Ana Cristina \\ Viana Campos, ${ }^{2}$ Mauro Henrique Nogueira Guimarães Abreu ${ }^{2}$ e \\ Efigênia Ferreira Ferreira²
}

Como citar

Palmier AC, Andrade DA, Campos ACV, Abreu MHNG, Ferreira EF. Indicadores socioeconômicos e serviços odontológicos em uma região brasileira desfavorecida. Rev Panam Salud Publica. 2012;32(1):22-9.

RESUMO Objetivo. Avaliar a associação entre proporção de exodontias, indicadores socioeconômicos e oferta de serviços odontológicos em uma região desfavorecida do Brasil.

Métodos. Um estudo ecológico foi realizado em 52 municípios do Estado de Minas Gerais, Brasil. Os indicadores socioeconômicos utilizados foram os critérios de alocação de recursos financeiros destinados à saúde, os índices de desenvolvimento humano municipal, de Theil e de Gini e as condições de saneamento. Em relação à oferta de serviços de saúde bucal, foram considerados o número de habitantes, o número de cirurgiões-dentistas residentes no município, o número de dentistas que trabalhavam no serviço público e o número de equipes de saúde bucal. A utilização de serviços foi avaliada pelos indicadores de saúde bucal preconizados pelo Pacto da Atenção Básica e pelo número de procedimentos realizados na atenção primária. As 17 variáveis avaliadas foram agrupadas em componentes. Analisou-se a relação entre esses componentes fatoriais e a variável dependente, proporção de exodontias.

Resultados. As variáveis foram agrupadas em seis componentes (condições socioeconômicas, desigualdades sociais, indicadores do Pacto da Atenção Básica, número de procedimentos realizados, razão dentistas:população, cobertura da Estratégia Saúde da Família) que explicaram, no conjunto, $73,5 \%$ da variância total. As desigualdades na distribuição de renda $(\mathrm{P}=0,031)$ e a cobertura da Estratégia Saúde da Familia $(\mathrm{P}=0,015)$ tiveram significativamente maior peso para explicar a diferença na proporção de exodontias realizadas nos municípios estudados.

Conclusões. Os fatores socioeconômicos e de organização dos serviços de saúde bucal explicam grande parte da mutilação dentária nessa região.

Palavras-chave Serviços de saúde bucal; assistência odontológica; desigualdades em saúde; fatores socioeconômicos; Brasil.

Artigo baseado na tese de doutorado da primeira autora, apresentada em 2011 à Universidade Federal de Minas Gerais (UFMG), Faculdade de Odontologia, Programa de Pós-Graduação em Odontologia Área de concentração: Saúde Coletiva.

2 Universidade Federal de Minas Gerais (UFMG), Faculdade de Odontologia, Departamento de Odontologia Social e Preventiva, Belo Horizonte (MG), Brasil. Correspondência: Mauro Henrique Nogueira Guimarães Abreu, maurohenriqueabreu@ig.com.br
Em 1986, 1996, 2003 e 2010, foram realizados no Brasil levantamentos epidemiológicos que mostraram uma grande redução nos índices de cárie em populações jovens ao longo do tempo. Em 1986,

UFMG, Faculdade de Odontologia, Belo Horizonte (MG), Brasil. o índice de dentes cariados, perdidos, obturados e danificados (CPOD) aos 12 anos era de 6,65 (1), contra 2,1 em 2010 (2).

Entretanto, em função do declínio restrito a algumas faixas etárias, a cárie dentária ainda é uma importante causa de dor, desordens funcionais e desconforto estético em grande parte da população. 
Além disso, a cárie é a principal causa de perdas dentárias, por si só um problema de saúde pública. Os resultados do levantamento epidemiológico nacional realizado em 2003 (3) mostraram que a perda dentária já ocorria precocemente aos 12 anos, embora em níveis mínimos (a média de dentes perdidos era igual a 0,18$)$. Com o aumento da idade, o componente perdido $(\mathrm{P})$ tornou-se mais prevalente, variando de 0,89 dos 15 aos 19 anos a 13,23 para a faixa dos 35 aos 44 anos e chegando até 25,83 , ou seja, quase a totalidade dos dentes, dos 65 aos 74 anos.

Por outro lado, os resultados preliminares da Pesquisa Nacional de Saúde Bucal SB Brasil 2010 (4) mostraram uma tendência de redução da cárie dentária e um aumento do acesso aos serviços odontológicos. Em 2003, os adultos de 35 a 44 anos de idade apresentavam um CPOD médio igual a 20,1, e o componente P correspondia a $65,72 \%$ do índice. Em 2010, o CPOD diminuiu para 16,3, assim como a proporção do componente $\mathrm{P}$, que chegou a 44,79\% (2). Apesar disso, a exodontia de dentes permanentes por cárie e doença periodontal ainda é uma das características mais marcantes da prática odontológica no Brasil (5).

A proporção de exodontias de dentes permanentes em relação às ações odontológicas básicas individuais foi avaliada em alguns estudos de abrangência local a partir das informações obtidas no Sistema de Informação Ambulatorial do Sistema Único de Saúde (SIA-SUS). No Estado de Pernambuco, a proporção de exodontias era maior em municípios de menor porte populacional, com baixa proporção da população cadastrada nas Equipes de Saúde Bucal (ESBs) e com relação de uma ESB para cada duas ou mais Equipes de Saúde da Família (6). Em Santa Catarina, fatores de organização do serviço de saúde bucal e condições sociais estiveram associados à proporção de exodontias (7). Nos três estados da Região Sul (Paraná, Santa Catarina e Rio Grande do Sul), Fischer et al. (8) identificaram que a proporção de exodontias foi maior quanto menor o índice de desenvolvimento humano (IDH) do município e quanto maior o índice de Gini e a cobertura das ESBs.

O Brasil é marcado por desigualdade socioeconômica, o que produz extensas consequências para a saúde, assim como para o acesso e a utilização de serviços odontológicos. O monitoramento dessas desigualdades é importante para a implementação de políticas públicas que busquem promover a saúde (9). Pela necessidade de reduzir as diferenças sociais existentes, o Sistema Único de Saúde (SUS), que adota como um dos seus princípios a equidade, busca tratar desigualmente os desiguais, investindo mais onde a carência é maior.

Ainda não há avaliações de abrangência nacional quanto ao efeito do serviço público odontológico sobre as desigualdades nos indicadores de saúde bucal, da mesma forma como são poucos os estudos aplicados a contextos regionais (10-12). Antunes e Narvai (13) argumentam que as estratégias de ampliação progressiva e o direcionamento dos recursos públicos de atenção odontológica, para fins programáticos, podem estar sendo bem-sucedidos quanto à redução das desigualdades em saúde, porém é escasso o conhecimento acerca dessas medidas em regiões caracterizadas por condições socioeconômicas desfavoráveis.

A realização de estudos que avaliem a associação entre indicadores socioeconômicos e indicadores de atenção primária à saúde bucal é importante para subsidiar a formulação de políticas de saúde que tenham como objetivo reduzir desigualdades em saúde, sobretudo no que diz respeito ao acesso e à utilização de serviços (7). Assim, o objetivo do presente estudo foi avaliar a associação entre proporção de exodontias, indicadores socioeconômicos e oferta de serviços odontológicos nos 52 municípios do Vale do Jequitinhonha, Minas Gerais, Brasil.

\section{MATERIAIS E MÉTODOS}

Foi realizado um estudo ecológico na bacia hidrográfica do rio Jequitinhonha, conhecida como Vale do Jequitinhonha, região mais pobre do Estado de Minas Gerais (14). A região é composta por 52 municípios, dividindo-se em Alto, Médio e Baixo Jequitinhonha. O Vale corresponde a $11,3 \%$ da área estadual total e apresenta uma população de aproximadamente 700000 habitantes, 3,6\% da população do estado. Localizada no nordeste de Minas Gerais, a região é um exemplo da persistência de iniquidades, com indicadores sociais (15) ainda desfavoráveis: alta taxa de mortalidade infantil (43,09 por 1000$)$, baixo nível educacional (média de 3,9 anos de estudo entre adultos) e alto percentual $(65,11 \%)$ da população vivendo com menos de meio salário mínimo mensalmente (16).

A variável dependente foi a proporção de exodontias de dentes permanentes em relação ao total de procedimentos odontológicos individuais realizados, por ser este considerado o pior desfecho em saúde bucal, indicando também o grau de mutilação relacionado à assistência odontológica individual (7, 8). Essa variável foi dicotomizada pela mediana da proporção de exodontias de dentes permanentes em relação ao total de procedimentos odontológicos individuais, que foi de $6,18 \%$. A partir dessa mediana, os municípios foram divididos em dois grupos: um com proporção de exodontias até $6,18 \%$ (grupo 1) e outro com proporção de exodontias acima de 6,18\% (grupo 2).

Foram considerados como variáveis independentes: os indicadores socioeconômicos, a oferta de serviços odontológicos, a utilização dos serviços públicos de saúde bucal e os indicadores construídos a partir dos procedimentos realizados pelo serviço público. Os indicadores socioeconômicos utilizados estão descritos na tabela 1 (15-18). A oferta de serviços odontológicos foi avaliada segundo a disponibilidade de profissionais nos municípios na tabela 2 (19-21).

A utilização dos serviços públicos de saúde bucal considerou os dados referentes à atenção primária disponíveis no SIA-SUS. Todos os dados são do ano de 2007. Foram considerados os procedimentos realizados na atenção primária, tendo sido registrado o número de procedimentos e os quatro indicadores do Pacto da Atenção Básica (IPABs) aprovados em 2006, que constituem um instrumento nacional de monitoramento das ações de saúde bucal referentes à atenção primária (22) (tabela 2). Além disso, foram analisados os procedimentos realizados na atenção primária em saúde bucal que constam na tabela de procedimentos do SIA-SUS para a construção de indicadores dos procedimentos realizados pelo serviço público (tabela 2) (19).

Os dados foram analisados no programa Statistical Package for the Social Sciences (SPSS), versão 18.0. Foi realizada a avaliação da normalidade das variáveis quantitativas por meio do teste de Kolmogorov-Smirnov e calculadas as medidas de tendência central e de variabilidade.

As variáveis independentes foram submetidas a análise fatorial explora- 
TABELA 1. Descrição das variáveis independentes: indicadores socioeconômicos, municípios do Vale do Jequitinhonha, Minas Gerais, Brasil, 2000

\begin{tabular}{|c|c|c|}
\hline Variável & Descrição & Valor \\
\hline Condições de habitação ${ }^{a}$ & $\begin{array}{l}\text { Domicílios com acesso à rede de água } \\
\text { Domicílios com acesso à rede de esgoto } \\
\text { Moradores em domicílios ligados à rede de água } \\
\text { Moradores em domicílios ligados à rede de esgoto }\end{array}$ & Percentual \\
\hline $\mathrm{IDH} \mathrm{H}^{\mathrm{b}, \mathrm{c}}$ & Longevidade, educação e renda & Escala de 0 a 1 \\
\hline Índice de Theil ${ }^{b}$ & Grau de desigualdade na distribuição de renda & Escala de 0 a $\infty$ \\
\hline Coeficiente de Gini ${ }^{a}$ & Grau de desigualdade segundo a renda domiciliar & Escala de 0 a 1 \\
\hline Fator de alocação de recursos ${ }^{d}$ & $\begin{array}{l}\text { Índice de necessidade em saúde (variáveis } \\
\text { epidemiológicas e socioeconômicas) + índice de porte } \\
\text { econômico (ICMS per capita) }\end{array}$ & Escala de 1 a 4 \\
\hline
\end{tabular}

a Fonte: Instituto Brasileiro de Geografia e Estatística, 2000 (15).

${ }^{b}$ Fonte: Programa das Nações Unidas para o Desenvolvimento, 2000 (16).

c IDH = Índice de desenvolvimento humano.

${ }^{d}$ Fonte: Fundação João Pinheiro, 2004 (17)

e Conforme Araújo (18).

tória. Essa análise estatística identifica agrupamentos de variáveis ou elementos através do menor conjunto possível de fatores comuns. O método utilizado foi o de componentes principais, extraindo-se os fatores com autovalores (eigenvalues) maiores que 1 e proporção de variância explicada pelos fatores comuns (comunalidades) superiores a 0,6. Após a extração, as comunalidades variam entre 0 e 1 , sendo 0 quando os fatores comuns não explicam nenhuma variância da va- riável e 1 quando explicam toda a sua variância (23).

O método Varimax foi utilizado para a rotação dos fatores retidos. A rotação é um procedimento empregado na análise fatorial com a finalidade de ajustar os fatores para melhorar a correlação entre as variáveis (itens) e o número de fatores, dando-lhes melhor significado interpretativo (24). A adequação dos dados para a análise fatorial foi demonstrada pelos testes de esfericidade de Bartlett
$(P \leq 0,001)$ e de Kaiser-Meyer-Olkin $(0,70)$. Foram coletadas 17 variáveis que permitiram a extração de seis componentes principais pela técnica de análise fatorial. O teste $t$ de Student foi utilizado para verificar a relação entre os componentes fatoriais formados e a variável dependente (proporção de exodontias), com significância estabelecida em $5 \%$.

Os dados obtidos são de domínio público e foram coletados do banco de dados do Banco de Dados do Sistema Único de Saúde (DATASUS).

\section{RESULTADOS}

A tabela 3 apresenta a estatística descritiva das variáveis estudadas segundo sua distribuição em quartis, dispersão e medidas de tendência central. Quanto ao porte populacional, 75\% dos municípios possuíam até 20030 habitantes, podendo ser considerados de pequeno porte. Em relação às condições de habitação, $62,17 \%$ ( $\pm 16,80 \%)$ da população residia em domicílio ligado à rede de água, mas apenas $30,48 \%( \pm 19,50 \%)$ dos moradores tinham suas residências ligadas à rede de esgoto. Todos os municípios foram classificados como tendo IDH médio. A distribuição da frequência do fator de

TABELA 2. Descrição das variáveis independentes: oferta e utilização de serviços odontológicos, municípios do Vale do Jequitinhonha, Minas Gerais, Brasil, 2007

\begin{tabular}{|c|c|c|c|}
\hline Variável & Indicador & Numerador $^{\mathrm{a}}$ & Denominador \\
\hline \multirow[t]{3}{*}{$\begin{array}{l}\text { Disponibilidade de profissionais } \\
\text { nos municípios }\end{array}$} & $\begin{array}{l}\text { Razão entre no. de habitantes e no. de } \\
\text { cirurgiões-dentistas residentes no } \\
\text { município }\end{array}$ & População total do município & No. de profissionais residentes ${ }^{b}$ \\
\hline & $\begin{array}{l}\text { Razão entre no. de habitantes e no. de } \\
\text { cirurgiões-dentistas cadastrados no } \\
\text { serviço público no município }\end{array}$ & População total do município & $\begin{array}{l}\text { No. de profissionais cadastrados no } \\
\text { serviço público }{ }^{c}\end{array}$ \\
\hline & $\begin{array}{l}\text { Razão entre no. de habitantes e no. de } \\
\text { Equipes de Saúde Bucal }\end{array}$ & População total do município & No. de Equipes de Saúde Bucal ${ }^{d}$ \\
\hline \multirow[t]{4}{*}{$\begin{array}{l}\text { Relativas ao Pacto da Atenção } \\
\text { Básica }\end{array}$} & $\begin{array}{l}\text { Cobertura da primeira consulta } \\
\text { programática }\end{array}$ & No. total de primeiras consultas no município & População total do municípioc \\
\hline & $\begin{array}{l}\text { Cobertura da ação coletiva Escovação } \\
\text { Dental Supervisionada }\end{array}$ & $\begin{array}{l}\text { Média mensal de participantes na ação } \\
\text { coletiva Escovação Dental Supervisionada }\end{array}$ & População total do municípioc \\
\hline & $\begin{array}{l}\text { Média de procedimentos odontológicos } \\
\text { básicos individuais }\end{array}$ & $\begin{array}{l}\text { No. de procedimentos odontológicos básicos } \\
\text { individuais }\end{array}$ & População total do município ${ }^{c}$ \\
\hline & $\begin{array}{l}\text { Proporção de procedimentos odontológicos } \\
\text { especializados em relação às ações } \\
\text { odontológicas individuais }\end{array}$ & $\begin{array}{l}\text { No. de procedimentos odontológicos } \\
\text { especializados }\end{array}$ & População total do municípioc \\
\hline \multirow[t]{2}{*}{$\begin{array}{l}\text { Construídas a partir dos } \\
\text { procedimentos realizados }\end{array}$} & $\begin{array}{l}\text { Proporção de procedimentos individuais } \\
\text { preventivos }\end{array}$ & $\begin{array}{l}\text { No. de procedimentos individuais } \\
\text { preventivos }^{e}\end{array}$ & $\begin{array}{l}\text { Total de procedimentos odontológicos } \\
\text { individuais }\end{array}$ \\
\hline & $\begin{array}{l}\text { Proporção de procedimentos individuais } \\
\text { restauradores }\end{array}$ & $\begin{array}{l}\text { No. de procedimentos individuais de } \\
\text { dentística básica }^{f}\end{array}$ & $\begin{array}{l}\text { Total de procedimentos odontológicos } \\
\text { individuais }\end{array}$ \\
\hline
\end{tabular}

a Dados obtidos do Banco de Dados do Sistema Único de Saúde (DATASUS), 2007 (19).

b Conforme Conselho Federal de Odontologia (20).

c Conforme DATASUS, 2007 (19).

d Conforme Secretaria de Estado da Saúde de Minas Gerais, 2007 (21)

e Fluorterapia, controle de placa, aplicação de selante, tartarectomia.

${ }^{\dagger}$ Restaurações plásticas diretas. 
TABELA 3. Estatística descritiva dos indicadores sociais, econômicos e de serviços municipais em 52 municípios do Vale do Jequitinhonha, Minas Gerais, Brasil, 2007

\begin{tabular}{|c|c|c|c|c|c|c|c|}
\hline Variável & Média & $\mathrm{DPa}$ & Mínimo & $\mathrm{P} 25^{\mathrm{b}}$ & Mediana & $P 75^{c}$ & Máximo \\
\hline População municipal (habitantes) ${ }^{d}$ & 13764 & 10198 & 2978 & 5726 & 10207 & 20030 & 44746 \\
\hline Domicílios com acesso à rede de água (\%) ${ }^{\mathrm{e}}$ & 63,31 & 16,13 & 26,82 & 51,41 & 67,81 & 76,17 & 89,03 \\
\hline População com acesso à rede de água (\%)e & 62,17 & 16,80 & 24,55 & 48,95 & 67,11 & 75,90 & 88,62 \\
\hline Domicílios com acesso à rede de esgoto $(\%)^{\mathrm{e}}$ & 31,46 & 19,71 & 0,52 & 12,20 & 30,48 & 47,78 & 62,88 \\
\hline População com acesso à rede de esgoto (\%)e & 30,48 & 19,50 & 0,41 & 12,32 & 29,09 & 46,68 & 62,49 \\
\hline Índice de Desenvolvimento Humano ${ }^{f}$ & 0,650 & 0,034 & 0,570 & 0,626 & 0,646 & 0,671 & 0,748 \\
\hline Índice de Theil ${ }^{\dagger}$ & 0,574 & 0,111 & 0,330 & 0,485 & 0,560 & 0,640 & 0,850 \\
\hline Índice de Gini ${ }^{\dagger}$ & 0,591 & 0,048 & 0,460 & 0,560 & 0,590 & 0,628 & 0,690 \\
\hline Fator de alocaçãog & 1,658 & 0,086 & 1,388 & 1,606 & 1,660 & 1,714 & 1,831 \\
\hline \multicolumn{8}{|l|}{ Razão entre no. de habitantes e no. de cirurgiões-dentistas cadastrados } \\
\hline no serviço público no municípiod & $1: 4226$ & $1: 2344$ & $1: 993$ & $1: 2666$ & $1: 3747$ & $1: 4928$ & $1: 14587$ \\
\hline \multicolumn{8}{|l|}{ Razão entre no. de habitantes e no. de cirurgiões-dentistas residentes } \\
\hline no município ${ }^{\mathrm{h}}$ & $1: 3278$ & $1: 2514$ & 0 & $1: 1646$ & $1: 2538$ & $1: 4427$ & $1: 14587$ \\
\hline Razão entre no. de habitantes e no. de Equipes de Saúde Bucal ${ }^{d}$ & $1: 5080$ & $1: 5445$ & 0 & $1: 2679$ & $1: 3514$ & $1: 6610$ & $1: 33061$ \\
\hline Cobertura da primeira consulta programática ${ }^{\mathrm{i}, \mathrm{j}}$ & 20,90 & 34,83 & 1,05 & 8,52 & 14,18 & 22,96 & 249,48 \\
\hline Cobertura da ação coletiva Escovação Dental Supervisionada ${ }^{i, j}$ & 18,01 & 29,79 & 0,00 & 0,30 & 7,50 & 24,76 & 170,08 \\
\hline Média de procedimentos odontológicos básicos individuais i,j & 1,11 & 1,04 & 0,12 & 0,43 & 0,76 & 1,42 & 6,14 \\
\hline \multicolumn{8}{|l|}{ Proporção de procedimentos odontológicos especializados em relação } \\
\hline às ações odontológicas individuais ${ }^{\mathrm{i}, \mathrm{j}}$ & 1,55 & 4,44 & 0,00 & 0,00 & 0,00 & 0,37 & 24,48 \\
\hline Proporção de procedimentos individuais preventivos ${ }^{i}$ & 52,06 & 19,04 & 0,00 & 41,26 & 56,57 & 64,46 & 88,20 \\
\hline Proporção de procedimentos de individuais restauradores ${ }^{i}$ & 26,86 & 13,37 & 0,00 & 19,19 & 27,60 & 33,94 & 57,55 \\
\hline Proporção de exodontias de dentes permanentes ${ }^{i}$ & 7,17 & 5,10 & 0,00 & 3,20 & 6,18 & 9,67 & 25,31 \\
\hline
\end{tabular}

\footnotetext{
a $\mathrm{DP}=$ desvio padrão.

b $\mathrm{P} 25=25^{\circ}$ percentil.

c $\mathrm{P} 75=75^{\circ}$ percentil.

d Banco de Dados do Sistema Único de Saúde (DATASUS), 2007 (19).

e Dados de População SIDRA/IBGE, 2000 (15).

f Programa das Nações Unidas para o Desenvolvimento, 2000 (16).

9 Fundação João Pinheiro, 2004 (17).

${ }^{\mathrm{h}}$ Conselho Federal de Odontologia e DATASUS, 2007 (19, 20).

i Sistema de Informação Ambulatorial do Sistema Único de Saúde (SIA-SUS), 2007.

j Critérios em que foram considerados 50 municípios por perda de dados em dois municípios.
}

alocação mostra que $44(84,6 \%)$ municípios pertenciam ao grupo 4, ou seja, apresentavam a maior necessidade em saúde e a menor capacidade de financiar com recursos próprios os cuidados com a saúde da população. Isso significa que um maior aporte de recursos financeiros era destinado a esses municípios. A distribuição dos índices de Theil e Gini mostra uma alta concentração de renda no grupo de municípios pesquisados, indicando desigualdade na distribuição de renda.

Quanto à presença de cirurgiões-dentistas, segundo os registros do Conselho Federal de Odontologia, dois municípios não apresentavam cirurgião-dentista residente, porém em todos havia profissionais cadastrados no serviço público. A razão entre o número de habitantes e o número de cirurgiões-dentistas contratados pelo serviço público nessa região apresentou desigualdade, variando de 1 cirurgião por 993 a 1 por 14587 habitantes. Em $75 \%$ dos municípios, a relação entre o número de dentistas e o número de habitante foi superior a 1 por 4 000. Ainda, dos 52 municípios da região, apenas oito $(15,4 \%)$ não apresentavam ESBs em 2007. Quanto à distribuição das ESBs, em $75 \%$ dos municípios existia 1 ESB para cada 6507 habitantes. A proporção média da população que teve acesso a uma consulta odontológica programática durante o ano de 2007 foi de $20,90 \%$.

$\mathrm{Na}$ análise fatorial exploratória, foram extraídos seis componentes (cada um formado por um grupo de variáveis) que explicaram, no conjunto, 73,5\% da variância total: condições socioeconômicas (componente 1), desigualdades na distribuição de renda (componente 2), IPABs (componente 3), procedimentos realizados (componente 4), razão entre dentistas e população (componente 5) e cobertura da Estratégia Saúde da Família (ESF) (componente 6). As denominações dos componentes foram atribuídas de forma discricionária, mas procurando representar o conjunto das variáveis associadas. As variáveis que tiveram maior peso em cada componente estão apresentadas na tabela 4 e organizadas por valor decrescente dos coeficientes rotacionados com a maior carga.
Fizeram sentido os valores negativos observados para as variáveis fator de alocação, no componente de condições socioeconômicas, procedimentos preventivos, no componente de procedimentos realizados, e razão entre número de ESBs e população do município, no componente de cobertura da ESF, já que essas variáveis deveriam se correlacionar negativamente, respectivamente, com os municípios onde as condições socioeconômicas fossem melhores, onde fossem realizados mais procedimentos restauradores e especializados e onde a cobertura da ESF fosse melhor.

Quanto maior a carga, mais a variável é uma pura medida do componente. Comrey e Lee (25) sugerem que cargas excedendo 0,71 (50\% de variância sobreposta) são excelentes; 0,63 (40\%) são muito boas; 0,55 (30\%) boas; 0,45 (20\%) regulares; e 0,32 (10\%) pobres. Como pode ser visto na tabela 2 , a maioria das cargas na solução rotacionada esteve acima de 0,65 , com exceção da variável razão entre número de habitantes e número de ESBs, cujo valor foi considerado pobre $(0,31)$. 
TABELA 4. Componentes rotacionados com a matriz de cargas fatoriais para os seis componentes extraídos, municípios do Vale do Jequitinhonha, Minas Gerais, Brasil, 2007

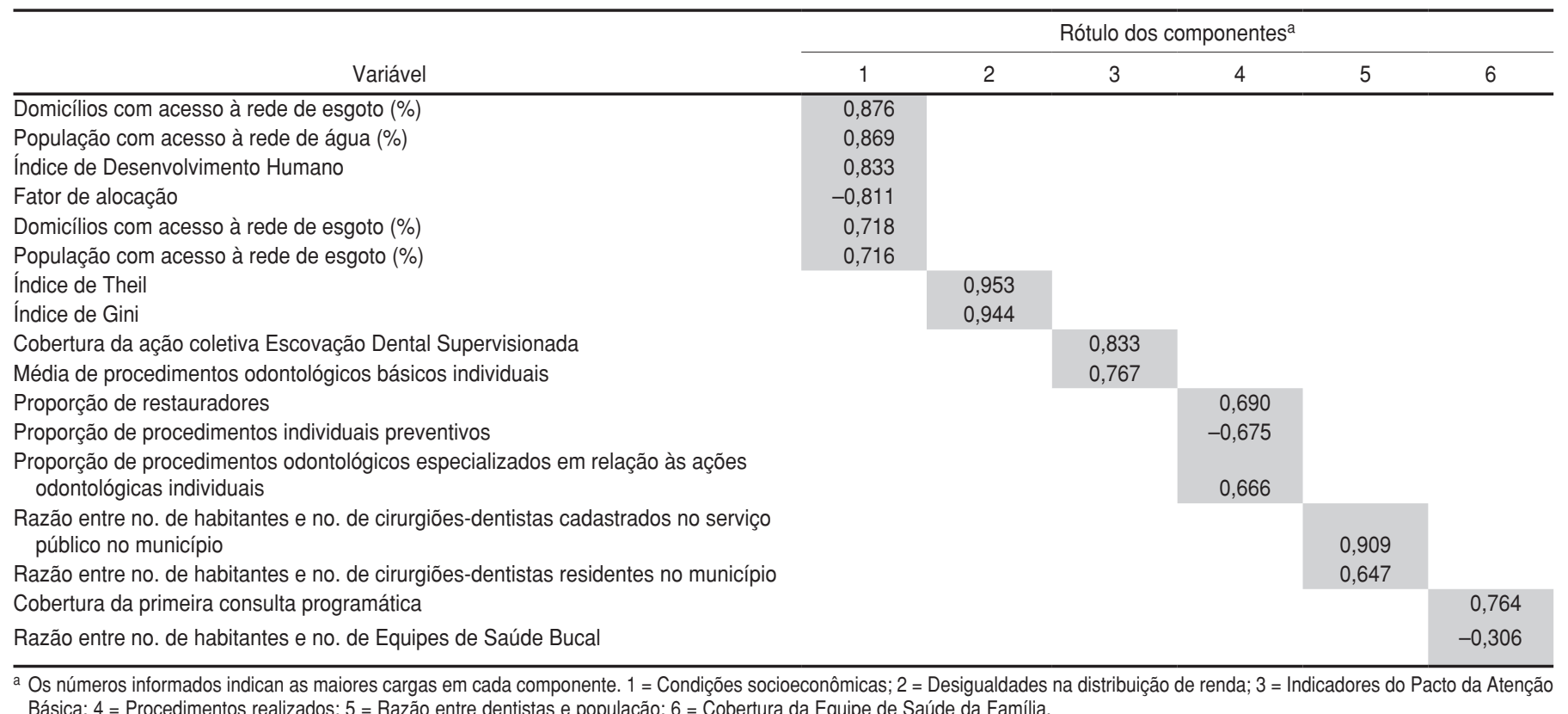

Para cada um dos seis componentes foi criada uma nova variável derivada das cargas fatoriais, expressa em escores padronizados (média de zero e desvio padrão de 1), indicando os desvios da média. As variáveis criadas pelo escore $\mathrm{Z}$ da análise fatorial foram utilizadas para testar as diferenças de cargas fatoriais entre os municípios com baixa proporção (grupo 1) e alta proporção (grupo 2) de exodontias.

As condições de desigualdades na distribuição de renda $(P=0,031)$ e a cobertura da $\operatorname{ESF}(P=0,015)$ tiveram significativamente maior peso para explicar as diferenças entre os municípios em relação à proporção de exodontias realizadas (tabela 5). Os municípios onde a proporção de exodontias foi maior possuíam maiores desigualdades na distribuição de renda e menor cobertura da ESF.

\section{DISCUSSÃO}

Os estudos ecológicos são considerados apropriados para investigar os efeitos do meio sobre a saúde de uma população, assim como para auxiliar na gestão de políticas públicas. Esses estudos permitem comparar os indicadores de situação de saúde e os indicadores socioeconômicos em diferentes áreas geográficas (26-28). Estudos ecológicos anteriores mostraram que a experiência de agravos bucais é suscetível às desigual- dades sociodemográficas e geográficas, sugerindo a existência de iniquidades em saúde bucal $(29,30)$, as quais se refletem, inclusive, no acesso e na utilização de serviços de saúde, conforme observado em estudos internacionais $(31,32)$ e nacionais $(8,33)$.

A distribuição de profissionais e o número de ESBs foram utilizados para avaliar a dimensão da oferta de serviços. Embora a Portaria 1 101, de 12 de junho de 2002, do Ministério da Saúde (que estabelece parâmetros assistenciais do SUS), sugira que a relação cirurgiãodentista:habitante pode variar de 1:1 500 a 1:5 000 (34), não há um número ideal estabelecido para essa relação, pois, para determinar tal proporção, é preciso levar em consideração elementos envolvidos no planejamento de recursos humanos odontológicos necessários em cada comunidade $(35,36)$. Na região estudada, foi encontrada uma relação de 1 cirurgião-dentista para cada 4226 habitantes. Os resultados mostraram que, embora nem todos os municípios tivessem dentistas residindo em seu território (dois municípios sem cirurgião-dentista residente), todos tinham profissionais cadastrados no serviço público. $\mathrm{O}$ fato de o profissional não residir no município onde trabalha pode gerar dois problemas: 1) os profissionais precisam viajar de um município para o outro para trabalhar; e 2) pode haver dificuldade no estabelecimento de vínculo profissional com a população adscrita.

Para aferir a utilização de serviços odontológicos, foram utilizados os IPABs e os procedimentos realizados na atenção primária em saúde bucal. A cobertura da primeira consulta odontológica é um dos indicadores principais que todos os municípios têm que pactuar. O município pode definir uma meta que deseja alcançar, dentro de sua realidade, com tendência crescente, visando à universalização. Para o ano de 2006, a Secretaria de Estado da Saúde de Minas Gerais considerou a cobertura de 12,52\% como parâmetro para esse indicador. Os resultados deste estudo mostraram que, em 75\% dos municípios do Vale do Jequitinhonha, a cobertura foi de $20,32 \%$, valor bem acima do esperado.

Os valores para a variável de desfecho (proporção de exodontias) não diferem dos encontrados em outros estudos realizados no Estado de Santa Catarina (7) e na Região Sul do país (8), ambos mais desenvolvidos e com condições socioeconômicas mais favoráveis. A escolha da proporção de exodontias é de grande importância para avaliar as práticas odontológicas que estão sendo realizadas. Os resultados mostraram que, dos seis componentes principais extraídos, apenas dois estavam associados à proporção de exodontias realizadas: desigualdades na distribuição de renda e cobertura da ESF. 
TABELA 5. Escores para cada um dos componentes derivados das cargas fatoriais para os municípios do grupo $1^{\mathrm{a}}(n=24)$ e do grupo $2^{\mathrm{b}}(n=26)$, municípios do Vale do Jequitinhonha, Minas Gerais, Brasil, 2007

\begin{tabular}{lrccc}
\hline & $\begin{array}{c}\text { Média } \\
\text { Componentes fatoriais/Exodontias }\end{array}$ & $\begin{array}{c}\text { Erro } \\
\text { padronizado }\end{array}$ & $\mathrm{t}^{\mathrm{c}}$ & $P$ \\
\hline Condições socioeconômicas & & & & \\
$\quad$ Grupo 1 & 0,040 & 0,221 & 0,268 & 0,790 \\
$\quad$ Grupo 2 & $-0,037$ & 0,184 & & \\
Desigualdades na distribuição de renda & $-0,314$ & 0,170 & $-2,220$ & $0,031^{\text {d }}$ \\
$\quad$ Grupo 1 & 0,290 & 0,209 & & \\
$\quad$ Grupo 2 & 0,207 & 0,232 & 1,421 & 0,162 \\
Indicadores do Pacto da Atenção Básica & $-0,191$ & 0,163 & & \\
$\quad$ Grupo 1 & $-0,130$ & 0,231 & $-0,878$ & 0,384 \\
$\quad$ Grupo 2 & 0,120 & 0,170 & & \\
Procedimentos realizados & & & \\
$\quad$ Grupo 1 & $-0,132$ & 0,107 & $-0,892$ & 0,377 \\
$\quad$ Grupo 2 & 0,121 & 0,254 & & \\
Razão entre dentistas e população & & & & \\
$\quad$ Grupo 1 & 0,353 & 0,255 & 2,527 & $0,015^{d}$ \\
$\quad$ Grupo 2 & $-0,326$ & 0,106 & & \\
$\quad$ Gobertura da Equipe de Saúde da Família & & &
\end{tabular}

a Grupo 1: municípios onde a proporção de exodontias é $\leq 6,18 \%$.

b Grupo 2: municípios onde a proporção de exodontias é > 6,18\%.

c Teste t de Student.

d $P<0,05$.

O primeiro é composto pelos índices de Theil e Gini, e o segundo, pelo percentual de cobertura da primeira consulta programática, ou seja, acesso a tratamento sequencial junto com a relação entre o número de ESBs e a população do município. Esses dados sugerem que o aumento da cobertura da assistência individual pode estar privilegiando outros tipos de procedimentos, podendo gerar mudanças nas condições de saúde bucal da população que está sendo atendida no serviço público. Em um estudo realizado no Estado de Pernambuco, observou-se que a proporção de exodontias em relação às ações odontológicas básicas individuais era menor onde a cobertura da população era maior $(P=$ $0,0027)$ e onde a relação ESB/população era mais favorável (6).

A ausência de associação entre o desfecho estudado e as outras variáveis socioeconômicas, como ocorreu em outros estudos (6-8), pode ser explicado pelo fato de os municípios apresentarem pouca variação socioeconômica, o que torna o universo homogêneo, com distribuição uniforme dos agravos.

Alguns estudos, também realizados em contextos regionais (10-12, 33, 37), mostraram que o planejamento de serviços públicos odontológicos apresentou uma tendência redistributiva ou próequidade, com maior provisão de recursos em municípios com piores indicadores socioeconômicos. Em Minas Gerais, o fator de alocação de recursos financeiros destinados à saúde foi desenvolvido em 2004 com o objetivo de promover uma maior equidade na distribuição geográfica de recursos financeiros nos municípios mineiros e permitir uma alocação equitativa de recursos, levando em consideração tanto a condição socioeconômica do município quanto as necessidades de saúde.

Os resultados de um estudo realizado pela Fundação João Pinheiro (14) mostraram que as mudanças ocorridas entre os valores municipais do Piso de Atenção Básica (PAB) per capita de 1997 e 2000 e as variáveis que refletem necessidades em saúde tenderam a beneficiar os municípios com maior necessidade em saúde, podendo ser consideradas próequidade. Isso sugere que a Secretaria de Estado da Saúde de Minas Gerais tem se empenhado em atender as diretrizes do Ministério da Saúde que buscam privilegiar municípios de pequeno porte populacional e baixo IDH, priorizando municípios com alto fator de alocação.

Uma das limitações do presente estudo é o fato de terem sido utiliza- dos dados secundários, em função da questionável consistência de registro dos mesmos. Um estudo realizado por Barros e Chaves (38) mostrou que o registro de procedimentos ambulatoriais da produção odontológica, como consultas, exodontias e restaurações, apresentou confiabilidade. Entretanto, os procedimentos coletivos apresentaram problemas de registro e padronização, o que comprometeu a confiabilidade dessas informações. É possível que haja sub ou supernotificação de procedimentos, como observado em dois municípios, que apresentaram coberturas acima de $100 \%$. Isso pode ser devido ao fato de o município atender, além de sua população, a de municípios vizinhos (7). $\mathrm{Ou}$, ainda, o registro dos procedimentos pode não corresponder à definição referida nos manuais do SIA-SUS, sendo computado o procedimento individual em vez do conjunto de procedimentos por indivíduo/mês. Também não foram avaliadas as condições de saúde da população nem a qualidade dos serviços prestados. A não avaliação da condição de saúde, da mesma forma que em outros estudos $(8,39)$, pode dificultar a compreensão mais completa das associações encontradas no presente estudo.

O Brasil está vivendo um momento de transição no que diz respeito à organização dos serviços de saúde bucal. Desde a implantação da odontologia na ESF em 2000, muitos avanços têm sido observados, principalmente depois de 2004, com a instituição da Política Nacional de Saúde Bucal. Os achados sugerem que as ESBs contribuíram para os resultados favoráveis dos indicadores, o que pode ser um indicativo de que os municípios do Vale do Jequitinhonha estão organizando a atenção em saúde bucal. Esperase que mais recursos sejam destinados às equipes e que os municípios ampliem sua rede de atenção básica, impactando positivamente na qualidade da saúde bucal da população. Para isso, é importante que haja adoção de políticas públicas de desenvolvimento social que ultrapassem o setor saúde, direcionando o benefício preferencialmente para as áreas onde haja maior necessidade, de forma a contribuir para a redução das desigualdades. Entretanto, são necessários mais estudos para avaliar o impacto dos serviços odontológicos nas condições de saúde bucal em regiões onde as condições socioeconômicas são desfavoráveis. 


\section{REFERÊNCIAS}

1. Brasil, Ministério da Saúde. Levantamento epidemiológico em saúde bucal: Brasil, zona urbana, 1986. Disponível em: http://dab. saude.gov.br/CNSB/vigilancia.php Acessado em 10 de março de 2011.

2. Roncalli AG. Projeto SB Brasil 2010: pesquisa nacional de saúde bucal revela importante redução da cárie dentária no país. Cad Saude Publica. 2011;27(1):4-5.

3. Brasil, Ministério da Saúde, Secretaria de Atenção à Saúde, Departamento de Atenção Básica, Coordenação Nacional de Saúde Bucal. Projeto SB Brasil 2003. Condições de saúde bucal da população brasileira $2002-$ 2003: resultados principais. Brasília: Ministério da Saúde; 2004.

4. Brasil, Ministério da Saúde. Pesquisa Nacional de Saúde Bucal - 2010. Nota à imprensa. Brasília: Ministério da Saúde; 2010. Disponível em: http://www.idisa.org.br/img/File/ SAUDE\%20BUCAL-NotaParaImprensa28dez2010\%20(2).pdf Acessado em 2 de maio de 2012.

5. Narvai PC, Antunes JLF. Saúde bucal: a autopercepção da mutilação e das incapacidades. Em: Lebrão ML, Duarte YAO, orgs. SABE: Saúde, Bem-Estar e Envelhecimento. O projeto SABE no município de São Paulo: uma abordagem inicial. Brasília: Organização Pan-Americana da Saúde; 2003. Disponível em: http://www.ciape.org.br/artigos/pro jeto_sabe.pdf\#page $=121$ Acessado em 5 de abril de 2011.

6. Pimentel FC. A atenção à saúde bucal no estado de Pernambuco: uma avaliação dos modelos assistenciais [dissertação]. Recife: Fundação Oswaldo Cruz; 2010. Disponível em: http://www.cpqam.fiocruz.br/bibpdf/ 2010pimentel-fc.pdf Acessado em 5 de abril de 2011.

7. Fernandes LS, Peres MA. Associação entre atenção básica em saúde bucal e indicadores socioeconômicos municipais. Rev Saude Publica. 2005;39(6):930-6.

8. Fischer TK, Peres KG, Kupek E, Peres MA. Indicadores de atenção básica em saúde bucal: associação com as condições socioeconômicas, provisão de serviços, fluoretação de águas e a estratégia de saúde da família no Sul do Brasil. Rev Bras Epidemiol. 2010;13(1):126-38.

9. Barros AJD, Bertoldi AD. Desigualdades na utilização e no acesso a serviços odontológicos: uma avaliação em nível nacional. Cienc Saude Coletiva. 2002;7(4):709-17.

10. Antunes JLF, Junqueira SR, Frazão P, Bispo CM, Pegoretti T, Narvai PC. City-level gender differentials in the prevalence of dental caries and restorative dental treatment. Health Place. 2003;9(3):231-9.

11. Antunes JLF, Pegoretti T, Andrade FP, Junqueira SR, Frazão P, Narvai PC. Ethnic disparities in the prevalence of dental caries and restorative dental treatment in Brazilian children. Int Dent J. 2003;53(1):7-12.

12. Souza TMS, Roncalli AG. Saúde bucal no Programa Saúde da Família: uma avaliação do modelo assistencial. Cad Saude Publica. 2007;23(11):2727-39.

13. Antunes JLF, Narvai PC. Políticas de saúde bucal no Brasil e seu impacto sobre as de- sigualdades em saúde. Rev Saude Publica. 2010;44(2):360-5.

14. Machado ENM, Fortes FBCTP, Somarriba M. Efeitos da introdução do PAB sobre a distribuição de recursos e a prestação de serviços: o caso de Minas Gerais. Cienc Saude Coletiva. 2004;9(1):99-111.

15. Instituto Brasileiro de Geografia e Estatística. Sistema IBGE de Recuperação Automática SIDRA. Disponível em: http://www.sidra. ibge.gov.br/ Acessado em 10 de março de 2011.

16. Programa das Nações Unidas para o Desenvolvimento. Atlas de Desenvolvimento $\mathrm{Hu}-$ mano no Brasil. Disponível em: http:/ / www. pnud.org.br/atlas/ Acessado em 2 de maio de 2012.

17. Fundação João Pinheiro. Índice de Necessidades de Saúde. Disponível em: http://www. fjp.gov.br/index.php/servicos/82-servicoscepp/55-indice-de-necessidade-em-saude Acessado em 2 de maio de 2012

18. Araujo MRN. Desafios contemporâneos à implantação da Saúde da Família em Minas Gerais. Tempus Acta Saude Coletiva. 2007;1(1):1-12. Disponível em: http://www. tempusactas.unb.br/index.php/tempus/ article/viewFile/387/370 Acessado em 10 de março de 2011

19. Departamento de Informática do SUS (DATASUS). Informações de saúde. Disponível em: http://www2.datasus.gov.br/DATA SUS/index.php?area $=02$ Acessado em 2 de maio de 2012

20. Conselho Federal de Odontologia. Relatório do CFO. Disponível em: http://cfo.org.br/ wp-content/uploads/2009/10/municipios brasil.pdf Acessado em 5 de dezembro de 2007.

21. Secretaria de Estado da Saúde de Minas Gerais, Coordenação de Saúde Bucal. Linha Guia. Belo Horizonte: SESMG; 2007. Disponível em: http://www.saude.mg.gov.br/ publicacoes/linha-guia/linhas-guia/Linha GuiaSaudeBucal.zip Acessado em 2 de maio de 2012

22. Brasil. Portaria 493/2006. Disponível em: http://dtr2001.saude.gov.br/sas/PORTA RIAS/Port2006/GM/GM-493.htm Acessado em 8 de março de 2012.

23. Hair JF, Black WC, Babin JB, Anderson RE, Tatham RL. Segmentation analysis. Em: Hair JF, Black WC, Babin JB, Anderson RE, Tatham RL, eds. Multivariate data analysis. 6th ed. Upper Saddle River: Prentice-Hall; 2009. Pp 427-81.

24. Coste J, Boué S, Ecosse E, Leplège A, Pouchot $\mathrm{J}$. Methodological issues in determining the dimensionality of composite health measures using principal component analysis: case illustration and suggestions for practice. Qual Life Res. 2005;14(3):641-54.

25. Comrey AL, Lee HB. A first course in factor analysis. 2nd ed. Hillsdale: Lawrence Erlbaum; 1992

26. Borrell C. Métodos utilizados no estudo das desigualdades sociais em saúde. Em: B arata RB, org. Condições de vida e situação de saúde. Rio de Janeiro: ABRASCO; 1997. Pp 167-95.
27. Peres MA, Antunes JLF. O método epidemiológico de investigação e sua contribuição para a saúde bucal. Em: Peres MA, Antunes JLF, eds. Epidemiologia da saúde bucal. Rio de Janeiro: Guanabara Koogan; 2006. Pp 3-18.

28. Tassinari WS, Leon AP, Werneck GL, Faerstein E, Lopes CS, Chor D. Contexto sócio-econômico e percepção da saúde bucal em uma população de adultos no Rio de Janeiro, Brasil: uma análise multinível. Cad Saude Publica. 2007;23(1):127-36.

29. Antunes JLF, Narvai PC, Nugent ZJ. Measuring inequalities in the distribution of dental caries. Community Dent Oral Epidemiol. 2004;32(1):41-8.

30. Baldani MH, Vasconcelos AGG, Antunes JLF. Associação do índice CPO-D com indicadores socioeconômicos e de provisão de serviços odontológicos no Estado do Paraná, Brasil Cad Saude Publica. 2004;20(1):143-52.

31. Hjern A, Grindefjord M, Sundberg H, Rosen M. Social inequality in oral health and use of dental care in Sweden. Community Dent Oral Epidemiol. 2001;29(3):167-74.

32. Nguyen L, Hakkinen U, Rosenqvist G. Determinants of dental service utilization among adults: the case of Finland. Health Care Manag Sci. 2005;8(4):335-45.

33. Junqueira SR, Araújo ME, Antunes JLF, Narvai PC. Indicadores socioeconômicos e recursos odontológicos em municípios do Estado de São Paulo, Brasil, no final do século XX. Epidemiol Serv Saude. 2006;15(4):41-53.

34. Brasil. Portaria 1 101/GM/2002. Disponível em: http://dtr2001.saude.gov.br/sas/ PORTARIAS/Port2002/Gm/GM-1101.htm Acessado em 8 de março de 2012.

35. Morita MC, Haddad AE, Araújo ME. Perfil atual e tendências do cirurgião-dentista brasileiro. Maringá: Dental Press; 2010. Disponível em: http://cfo.org.br/wp-content/ uploads/2010/04/PERFIL_CD_BR_web.pdf Acessado em 8 de março de 2012.

36. Viacava F, Almeida C, Caetano R, Fausto M, Macinko J, Martins M, et al. Uma metodologia de avaliação do desempenho do sistema de saúde brasileiro. Cienc Saude Coletiva. 2004;9(3):711-24.

37. Baldani $\mathrm{MH}$, Brito $\mathrm{WH}$, Lawder JAC, Mendes YBE, Silva FFM, Antunes JLF. Determinantes individuais da utilização de serviços odontológicos por adultos e idosos de baixa renda. Rev Bras Epidemiol. 2010;13(1):150-62.

38. Barros SG, Chaves SCL. A utilização do Sistema de Informações Ambulatoriais (SIA-SUS) como instrumento para a caracterização das ações de saúde bucal. Epidemiol Serv Saude. 2003;12(1):41-51.

39. Baldani MH, Almeida ES, Antunes JLF. Serviços públicos odontológicos: provisão e equidade no Estado do Paraná, Brasil. Rev Saude Publica. 2009;43(3):446-54.

Manuscrito recebido em 31 de maio de 2011. Aceito em versão revisada em 6 de dezembro de 2011. 
ABSTRACT Objective. To evaluate the association between the proportion of tooth extractions, socioeconomic indicators, and the availability of oral health services in an underprivileged area of Brazil.

Socioeconomic indicators Methods. An ecological study was carried out in 52 municipalities in the state of and oral health services in an underprivileged area of Brazil Minas Gerais, Brazil. The socioeconomic indicators employed were criteria for health care resources allocation, municipal human development index, Theil index, Gini coefficient, and sanitation conditions. Concerning the availability of oral health services, the following were considered: number of inhabitants, number of dental surgeons living in the city, number of dentists working in the public services, and number of municipal oral health care teams. The utilization of oral health services was evaluated using the indicators recommended by the Health Ministry's Basic Care Package (Pacto da Atencão Básica in Portuguese) and the number of procedures carried out in the primary care setting. The 17 variables assessed were grouped into factorial components, which were then analyzed in terms of their relationship with the dependent variable, tooth extractions.

Results. The following six components explained $73.5 \%$ of the overall variance: socioeconomic conditions, social inequality, Basic Care Package indicators, number of procedures carried out, ratio of dentists to inhabitants, and coverage of the Family Health Strategy. Inequalities in income distribution $(P=0.031)$ and coverage by the Family Health Strategy $(P=0.015)$ contributed significantly to explain the difference in the proportion of tooth extractions in the different municipalities under study.

Conclusions. The dental loss observed in the region is largely explained by socioeconomic factors and aspects related to the organization of oral health services.

Key words Dental health services; dental care; health inequalities; socioeconomic factors; Brazil. 